\title{
INTERVENÇÃO EDUCATIVA NOS CUIDADOS DA DERMATITE DA FRALDA EM IDOSOS INSTITUCIONALIZADOS
}

\author{
Aline Teixeira Alves ${ }^{1}$ \\ Raquel Henriques Jácomo² \\ Wellington Alves Epaminondas ${ }^{3}$ \\ Dayanne Soares Faria ${ }^{4}$ \\ Renata de Menezes Leite ${ }^{5}$ \\ Liana Barbaresco Gomide ${ }^{6}$ \\ Adriano Bueno Tavares ${ }^{7}$
}

\footnotetext{
1 Graduada em Fisioterapia. Doutora em Ciência da Saúde. Professora Adjunta da Universidade de Brasília (UnB) vinculada ao colegiado do curso de Fisioterapia. E-mail: aline.urogineco@gmail.com 2 Graduada em Fisioterapia. Mestre em Ciências Médicas. Fisioterapeuta do Hospital Universitário de Brasília (HUB). E-mail: raquel.jacomo@gmail.com

3 Graduado em Medicina. Especialista em Urologia. Médico do Hospital Regional da Asa Norte (HRAN). E-mail: drwellington.urobrasilia@hotmail.com

4 Graduada em Fisioterapia. Fisioterapeuta do Studio Pilates Larissa Carvalho. E-mail: dayanne. soares@yahoo.com

5 Graduada em Fisioterapia. Fisioterapeuta da Fisiotrauma Clínica de Fisioterapia. E-mail: rezinham|@ hotmail.com

6 Graduada em Fisioterapia. Doutora em Ciências Médicas. Professora Adjunta da Universidade de Brasília (UnB) vinculada ao colegiado do curso de Fisioterapia. E-mail: lianagomide@unb.br

7 Graduado em Medicina. Doutor em Tocoginecologia. Professor Adjunto da Universidade Católica de Brasília (UCB) vinculado ao Departamento de Gerontologia. E-mail: atavares@pos.ucb.br
} 
resumo

\begin{abstract}
Este estudo teve por objetivo analisar a efetividade da intervenção educativa na prevenção e tratamento da dermatite da fralda de idosos institucionalizados. Trata-se de um estudo qualitativo, longitudinal e prospectivo a partir de uma amostra de 38 idosos. $\bigcirc$ instrumento foi composto por uma cartilha, entregue aos agentes de saúde, na qual ela apresentava medidas para minimizar os problemas da dermatite. Foi realizada uma avaliação clínica da dermatite da fralda antes e após a aplicação das medidas. Verificou-se que apenas 7 idosos com dermatite leve persistiram com o quadro. Vinte obtiveram a cura dos sintomas, representando, assim, 81,58\% dos idosos. Houve também uma diminuição dos sintomas, na qual a descamação foi representada por 93,33\% de melhora, a hiperemia por 77,41\% e o edema por $93,75 \%$. Diante dos resultados deste estudo, constatou-se que as medidas propostas foram adequadas e eficazes no tratamento da dermatite de grau leve em idosos institucionalizados.
\end{abstract}

\title{
palavras-chave
}

Exantema. Incontinência Urinária. Saúde do Idoso Institucionalizado. Promoção da Saúde.

\section{Introdução}

O trato geniturinário, assim como outros sistemas, é influenciado pelos efeitos interativos e aditivos das mudanças ocasionadas pelo envelhecimento. Embora muitos idosos se conservem ativos e saudáveis, estudos mostram que a incidência de disfunções vesicais vem aumentando com a idade (CLEMENS et al., 2011). A bexiga passa por alterações importantes com o avanço da idade, sendo a diminuição da capacidade vesical a principal delas. Com isso, queixas como incontinência urinária, dificuldade de urinar, urgência e urge-incontinência, noctúria e aumento da frequência urinária tornam-se frequentes em idosos, principalmente nos institucionalizados. Estima-se que 30\% dos pacientes em hospitais e $50 \%-70 \%$ de pacientes residentes em instituições de longa permanência (ILP) apresentam incontinência urinária (IU) (WEISS, 1998).

A incontinência urinária associada à dermatite irritativa da fralda (DIF) tem sido muito relatada nos últimos anos (ZAHORSKY et al., 1915; GRAY et al., 2007). Também conhecida como "dermatite da área da fralda irritativa primária", 
a DIF é interpretada como o resultado final de uma cascata de eventos desencadeados, inicialmente, por lesões no nível do estrato córneo induzidas por exposição a múltiplos fatores, tais como hiperidratação, fricção, temperatura, irritantes químicos, urina e fezes (BERG; BUCKINGHAM; STEWART, 1986; PRASAD; SRIVASTAVA; VERMA, 2003).

A verdadeira prevalência não é conhecida, porém alguns estudos epidemiológicos estimam que $5,6 \%$ até $50 \%$ das pessoas incontinentes usuárias de fralda ou absorventes apresentam a DIF (GRAY et al., 2007). Um estudo japonês identificou uma prevalência de $17 \%$ em pacientes idosos incontinentes (SHIGETA et al., 2009).

Os princípios do tratamento consistem na escolha adequada da fralda, na frequência de trocas, na limpeza e no uso de produtos de barreira (óxido de zinco e petrolato). Apesar destas práticas de cuidados preventivos, a deterioração da pele ocorre em pessoas idosas em razão da diminuição da função de barreira da pele, relacionada ao envelhecimento, e, assim, reduz a resistência da pele à exposição à umidade (COOPER; GRAY, 2001; LEWIS-BYERS; THAYER; KAHL, 2002; BLISS et al., 2007).

Os cuidados paliativos, embora vinculados intimamente ao envelhecimento, propõem técnicas que, numa perspectiva humana, permitam aliviar os desgastes físicos e psíquicos, sendo necessária uma equipe interdisciplinar para a aplicação das mesmas.

O presente estudo tem como finalidade analisar a efetividade da intervenção educativa na prevenção e no tratamento da dermatite da fralda de idosos institucionalizados por meio de uma cartilha de cuidados da dermatite da fralda em idosos.

\section{Materiais e métodos}

Trata-se de um estudo quantitativo, longitudinal e prospectivo realizado em uma ILP localizada na cidade de Brasília, Distrito Federal. Esta ILP atende 150 idosos distribuídos em 3 alas (ala A, com 58 do sexo feminino; ala B, com 62 do sexo masculino; e a ala $\mathrm{C}$, com 30 idosos acamados de ambos os sexos).

Foram incluídos no estudo todos agentes de saúde que trabalham na referida ILP no período proposto para a coleta de dados. Deles, 8 eram técnicos em enfermagem e 14, cuidadores de idosos. Todos foram treinados para realizar a intervenção com os idosos da instituição que utilizavam fralda, independente do gênero e do nível de funcionalidade. Desta forma, 38 idosos, sendo 20 mulheres e 18 homens, foram submetidos ao processo de intervenção. 
O instrumento utilizado como intervenção foi uma cartilha, na qual havia orientações para minimizar os problemas da dermatite da fralda relacionados aos idosos. As medidas propostas foram: trocar as fraldas a cada 4 horas, utilizar banheiro ou micção no leito com recipientes, aparar os pelos pubianos, realizar a higiene da região perineal a cada vez que os idosos urinassem ou evacuassem, aplicar pomada com óxido de zinco (Hipoglós@) uma vez por dia, orientar os idosos a avisar os agentes de saúde quando aqueles estivessem molhados, trocar o lençol quando molhados, adequar os tamanhos das fraldas, ingerir no mínimo 1 litro de líquido por dia, evitar líquidos 1 hora antes de dormir e expor a área da dermatite ao sol.

Um único médico urologista, colaborador da pesquisa, realizou a avaliação clínica da dermatite da fralda em todos os idosos usuários de fraldas residentes na ILP, antes e após três semanas de intervenção, de forma cega (não foi informado sobre o procedimento educacional). Durante a segunda avaliação, o urologista não teve acesso à avaliação inicial do idoso. Foram avaliados os seguintes sinais: descamação, hiperemia, edema, disúria, úlcera e infecções secundárias.

Esta pesquisa foi submetida e aprovada pelo Comitê de Ética em Pesquisa do Centro Universitário de Brasília (UniCEUB), obtendo parecer favorável para sua realização (protocolo 2893.0.000.303 - 08). Os agentes da saúde participantes do estudo assinaram o Termo de Consentimento Livre e Esclarecido.

Foi utilizado, para análise estatística, o software Microsoft Office Excel 2007, com avaliação da melhora em percentual dos sintomas da dermatite da fralda, antes e após a intervenção.

\section{Resultados}

A ILP abordada neste estudo abriga 150 idosos, sendo que 38 idosos usavam fralda, o que corresponde a 25,3\% consistindo em 20 mulheres e 18 homens com média de idade de 80,14 anos $( \pm 10,48)$. Destes idosos usuários de fraldas, apenas 10 não estavam restritos ao leito e os outros 28 encontravam-se na ala C da ILP, pois apresentavam restrição ao leito.

O médico urologista, ao realizar a avaliação clínica em todos os idosos usuários de fraldas, comprovou a presença da dermatite da fralda nos 38 idosos (25,3\%), observando que destes, 15 (39,4\%) apresentavam descamação; 31 (81,5\%), hiperemia; 16 (42,1\%), edema; e nenhum mostrou as características clínicas de disúria, úlceras e infecções secundárias. Vale ressaltar que todos os idosos apresentaram dermatite em grau leve. 
Todos os idosos usuários de fraldas foram reavaliados na ILP após o período de 3 semanas, na qual os agentes de saúde aplicaram as medidas propostas na cartilha, observando que o número de idosos que apresentaram a descamação diminuiu para $1(2,6 \%)$; o dos que encontravam-se com hiperemia, para $7(18,4 \%)$; e, dos tinham edema, apenas $1(2,6 \%)$ persistiu com este sintoma, comprovando a adesão dos agentes de saúde em relação às medidas propostas na cartilha. Contudo, todos continuaram com o quadro clínico de incontinência urinária, já que não houve intervenção abordando-a.

De uma maneira geral, dos 38 idosos que apresentaram a dermatite da fralda em grau leve, apenas 7 persistiram com o quadro e 20 obtiveram a cura dos sintomas da dermatite, representando $81,58 \%$. De acordo com a proporção das características clínicas avaliadas antes e após a aplicação das medidas propostas na cartilha, foi possível observar em todos os idosos avaliados que o quadro clínico de incontinência urinária persistiu em $100 \%$ dos casos; dos $39,4 \%$ que tinham inicialmente descamação, apenas 2,6\% persistiram com o sintoma; $81,5 \%$ apresentavam hiperemia, sendo que $18,4 \%$ continuaram com o sintoma; e dos $42,1 \%$ que se encontravam com o edema, apenas $2,6 \%$ persistiram com quadro clínico. Foi possível observar, de acordo com a diferença do número de idosos com as características clínicas da dermatite da fralda na avaliação com a reavaliação, uma melhora da descamação, da hiperemia e do edema, correspondendo a 93,3\%, 77,4\% e 93,7\%, respectivamente.

\section{Discussão}

O presente estudo trabalhou com a hipótese de que as condições de pele dos idosos melhorassem após a intervenção com a cartilha de orientações para DIF ou que a DIF fosse evitada com este tipo de instrumento. Observou-se que a cartilha acelerou o tratamento da DIF, com melhora significativa do quadro dos pacientes.

Uma das principais características do processo do envelhecimento é a diminuição das funções fisiológicas e orgânicas, o que gera o aparecimento de problemas relacionados à saúde como incontinência urinária, noctúria, urgência e urge-incontinência e infecções. Com isso, o aparecimento da DIF se torna facilitado, já que grande parte dos paceinetes incontinentes utilizam fraldas ou absorventes.

Na literatura, existem estudos que avaliam os vários tipos de fraldas e absorventes como tratamento da dermatite (BEGUIN et al., 2010; SUGAMA et al., 2012). Porém, apenas cuidados paliativos e troca dos tipos de absorventes 
se tornam ineficazes no combate à DIF. Por este motivo, torna-se importante o treinamento e o acompanhamento dos agentes de saúde para estes realizarem os cuidados específicos.

Estudos randomizados e controlados investigando o efeito de programas de cuidado da pele já foram previamente conduzidos e todos mostraram que as medidas educativas são eficazes e fundamentais para a prevenção e o tratamento dos problemas de pele (COOPER; GRAY, 2001; LEWIS-BYERS; THAYER; KAHL, 2002; BLISS et al., 2007). Uma revisão de literatura concluiu que mudanças frequentes de fraldas, o uso de fraldas superabsorventes e a proteção da pele perineal com um produto que contenha óxido de vaselina ou zinco são eficazes no tratamento da dermatite da fralda (HEIMALL et al., 2012), assim como que, principalmente, tanto uma educação contínua como o acompanhamento por parte dos cuidadores são necessários para melhorar a prevenção e o tratamento das dermatites. Porém, a revisão de Heimall et al. focou em dermatites das fraldas em crianças.

De acordo com Brasil (2002), as propostas de elaboração de cartilhas específicas procuram reproduzir, da melhor forma possível, as orientações sobre os cuidados específicos com a população desejada, tornando mais acessíveis aos agentes de saúde as informações básicas para a promoção e manutenção da saúde.

De acordo com o Caderno de Atenção Básica: Envelhecimento e Saúde da Pessoa Idosa, a IU está presente em 25\%-30\% dos idosos institucionalizados (BRASIL, 2006), dados que se confirmam no resultado do presente estudo, no qual 25,3\% dos idosos residentes na ILP em questão apresentaram a incontinência urinária. O estudo de Foureur et al. (2006) mostrou que este é um distúrbio comum da pele de idosos e frequente em pacientes acamados, sendo decorrente dos agentes irritantes no meio ambiente com a fralda como fricção, oclusão, umidade, maceração, urina e enzimas lipolíticas e proteolíticas fecais.

Foi possível constatar, com este estudo, que a proporção de dermatite em idosos usuários de fralda que residem em ILP é alta e que os sintomas de maior prevalência foram a IU, a hiperemia, a descamação e o edema. O presente estudo vai de acordo com o de Gray (2007), que descreveu uma prevalência de $24 \%$ em pacientes idosos institucionalizados.

O que pode justificar a magnitude dos resultados por um curto período de tempo é o fato dos idosos avaliados apresentaram a dermatite da fralda num estágio de leve a moderado, respondendo, assim, facilmente ao tratamento. 
Entre as limitações do presente estudo, podemos citar a escassez de instrumentos de avaliação. Como não existe nenhum questionário validado ou nenhuma forma de avaliação padronizada para a avaliação da DIF, o estudo manteve-se descritivo, avaliando os principais sintomas. Sugerem-se outros estudos abordando outras ILP para confirmar estes resultados e avaliar os efeitos deste instrumento na melhoria clínica da DIF nos pacientes idosos institucionalizados.

\title{
5 Considerações finais
}

As medidas propostas na cartilha para promoção e manutenção da saúde do idoso foram adequadas e eficazes no tratamento da dermatite em grau leve de idosos institucionalizados.

\author{
EDUCATIONAL INTERVENTION \\ IN THE CARE OF DIAPER DERMATITIS \\ IN INSTITUTIONALIZED ELDERLY
}

\section{abstract}

This study aimed to examine the effectiveness of educational interventions in the prevention and treatment of diaper dermatitis in the institutionalized elderly. This is a prospective, qualitative and longitudinal study from a sample of 38 old people. The instrument consisted of a booklet given to health workers with instructions to minimize the problems of dermatitis. We performed a clinical assessment of diaper dermatitis before and after implementing the measures. It was found that only 7 elderly patients with mild dermatitis persisted in the frame. Twenty of them obtained the cure of symptoms, representing $81.58 \%$ of the elderly. There was also a decrease of symptoms, in which the improvement of peeling was represented by $93.33 \%$, hyperemia by $77.41 \%$, and edema by $93.75 \%$. According to the results of this study, the proposed measures are appropriate and effective in the treatment of mild dermatitis in the institutionalized elderly.

keywords

Exanthema. Urinary Incontinence. Health of Institutionalized Elderly. Health Promotion. 
BEGUIN, Anne-Marie et al. Improving diaper design to address incontinence associated dermatitis. BMC Geriatrics, London, v. 10, p. 86, Nov. 2010.

BERG, Ronald Wayne; BUCKINGHAM, Kent W.; STEWART, Robert L. Etiologic factors in diaper dermatitis: the role of urine. Pediatric Dermatology, Boston, v. 3, n. 2, p. 102-106, Feb. 1986

BLISS, Donna Zimmaro et al. An economic evaluation of four skin damage prevention regimens in nursing home residents with incontinence. Journal of Wound, Ostomy and Continence Nursing, St. Louis, v. 34, n. 2, p. 143-152, Mar./Apr. 2007.

BRASIL. Ministério da Saúde. Secretaria de Políticas de Saúde. Projeto Promoção da Saúde. As Cartas da Promoção da Saúde. Brasília: Ministério da Saúde, 2002. 56 p.: il. Série B. Textos Básicos em Saúde.

Ministério da Saúde. Secretaria de Políticas de Saúde. Guia prático do cuidador. Brasília: Ministério da Saúde, 2006. 192 p.: il. Série A. Caderno de Atenção Básica no19.

CLEMENS, Quentin James et al. Perceptions of "urgency" in women with interstitial cystitis/bladder pain syndrome or overactive bladder. Neurourology \& Urodynamics, New York, v. 30, n. 3, p. 402-405, Mar. 2011.

COOPER, Pam; GRAY, David. Comparison of two skin care regimes for incontinence. British Journal of Nursing, London, v. 10, p. 6-20, Mar. 2001. Supplement 1.

FOUREUR, Nicolas et al. Prospective aetiological study of diaper dermatitis in the elderly. British Journal of Dermatology, London, v. 155, n. 5, p. 941-946, Nov. 2006.

GRAY, Mikel et al. Incontinence-associated dermatitis: a consensus. Journal of Wound, Ostomy and Continence Nursing, St. Louis, v. 34, n 1, p. 45-54, Jan./Feb. 2007.

HEIMALL, Lauren Marie et al. Beginning at the bottom: evidence-based care of diaper dermatitis. The American Journal of Maternal/Child Nursing, New York, v. 37, n 1, p. 10-16, Jan./Feb. 2012.

LEWIS-BYERS, Kari; THAYER, Debra; KAHL, Ann. An evaluation of two incontinence skin care protocols in a long-term care settings. Ostomy Wound Management, King of Prussia, PA, v. 48, n. 12, p. 44-51, Dec. 2002.

PRASAD, Hindi Rajendra; SRIVASTAVA, Pushplata; VERMA, Kaushal Kumar. Diaper dermatitis-an overview. Indian Journal of Pediatrics, New Delhi, v. 70, n. 8, p. 635-637, Aug. 2003.

SHIGETA, Yoshie et al. Exploring the relationship between skin property and absorbent pad environment. Journal of Clinical Nursing, Oxford, v. 18, n. 11, p. 1607-1616, June 2009.

SUGAMA, Junko et al. Efficacy of an improved absorbent pad on incontinence-associated dermatitis in older women: cluster randomized controlled trial. BMC Geriatrics, London, v. 12, p. 22, May 2012.

WEISS, Barry David. Diagnostic Evaluation of Urinary Incontinence in Geriatric Patients. American Family Physician, Kansas City, v. 57, n. 11, p. 2675-2684, June 1998.

ZAHORSKY, John. The ammoniacal diaper in infants and young children. The American Journal of Diseases of Children, Chicago, v. 10, n. 6, p. 436-444, Dec. 1915. 